\title{
Genotypic and haplotypic associations of the $D B H$ gene with plasma dopamine $\beta$-hydroxylase activity in African Americans
}

\author{
Yi-lang Tang ${ }^{1,5}$, Michael P Epstein ${ }^{2}$, George M Anderson ${ }^{3}$, Cyrus P Zabetian ${ }^{4}$ and \\ Joseph F Cubells*,1,5
}

\begin{abstract}
${ }^{1}$ Department of Psychiatry, Yale University School of Medicine and VA Connecticut Healthcare System, New Haven, CT, USA; ${ }^{2}$ Department of Human Genetics, Emory University, Atlanta, GA, USA; ${ }^{3}$ Child Study Center, Yale University School of Medicine, New Haven, CT, USA; ${ }^{4}$ Department of Neurology, University of Washington School of Medicine and Geriatric Research Education and Clinical Center, VA Puget Sound Health Care System, Seattle, WA, USA
\end{abstract}

Several variants at $D B H$ are significantly associated with plasma $D \beta H$ activity $(p D \beta H)$. However, the overwhelming majority of data on this genotype-phenotype relationship has been gathered in samples from Europeans and European Americans (EAs). In this study, we examined the relationship between $D B H$ polymorphisms and $\mathrm{pD} \beta \mathrm{H}$ in samples from African-American (AA) subjects. Genotypes were determined at a 19-bp insertion/deletion polymorphism (ins/del) and four single-nucleotide polymorphisms (SNPs) at DBH in 109 samples. Analyses were performed using analyses of variance (ANOVAs) (for individual SNPs) and regression procedures (to assess the joint effects and the specific SNP-based haplotypes). We found: (1) single-variant analysis of all polymorphisms revealed apparent associations to $\mathrm{pD} \beta \mathrm{H}$, with rs 1611115 accounting for the largest proportion of the variance in $\mathrm{pD} \beta \mathrm{H}(28.7 \%)$ and ins/del the smallest $(6.5 \%) ;(2)$ modest but significant linkage disequilibrium (LD) existed between ins/del and rs1611115; (3) LD between all other pairs of variants was not observed; (3) stepwise regression showed that a model containing rs 1611115 , rs 2519152 and rs6271 accounted for $37.6 \%$ of the variance in $\mathrm{pD} \beta \mathrm{H}$, with rs6271 showing additional $7.6 \%$ above the effect of rs 1611115 , and $\mathrm{rs} 2519152$ showing additional $2 \%$ above rs 1611115 and rs6271; (4) two common haplotypes, C-T-C and T-C-C at rs1611115-rs2519152-rs6271 were significantly associated with $\mathrm{pD} \beta \mathrm{H}(P=0.0025$ and 0.0036 , respectively). The data support the validity of prior reported associations and underscore the importance of analyzing multiple SNPs across $D B H$ in future association studies examining disease and biochemical phenotypes.

European Journal of Human Genetics (2007) 15, 878-883; doi:10.1038/sj.ejhg.5201838; published online 25 April 2007

Keywords: dopamine $\beta$-hydroxylase; African American; linkage disequilibrium; genetic association; haplotype

\footnotetext{
*Correspondence: Dr JF Cubells, Department of Human Genetics, Emory University, 615 Michael Street, Suite 301, Atlanta, GA 30322, USA. Tel: +1 404727 2005; Fax: +1 404727 5764;

E-mail: jcubells@genetics.emory.edu

${ }^{5}$ Current address: Departments of Human Genetics and Psychiatry and Behavioral Sciences, Emory University School of Medicine, Atlanta, GA, USA.

Received 18 October 2006; revised 23 February 2007; accepted 22 March 2007; published online 25 April 2007
}

\section{Introduction}

Dopamine (DA) $\beta$-hydroxylase $(\mathrm{D} \beta \mathrm{H})$ catalyzes the conversion of DA into norepinephrine (NE) and is localized within vesicles of central noradrenergic and adrenergic neurons as well as peripheral noradrenergic (sympathetic) neurons and adrenomedullary neurosecretory cells. ${ }^{1} \mathrm{D} \beta \mathrm{H}$ enzyme activity and $\mathrm{D} \beta \mathrm{H}$ immunoreactive protein are measurable in the serum (or plasma) because the enzyme is released from vesicles during sympathetic activity. ${ }^{2-4}$ 
Plasma $\mathrm{D} \beta \mathrm{H}$ activity $(\mathrm{pD} \beta \mathrm{H})$ is a genetic trait controlled by only a few genes. ${ }^{1,5}$ Most of the variance in $\operatorname{pD} \beta \mathrm{H}$ is explained by variation in plasma levels of $\mathrm{D} \beta \mathrm{H}$ protein, as shown by strong correlations $(r \geqslant 0.80)$ between $\mathrm{pD} \beta \mathrm{H}$ and plasma levels of $\mathrm{D} \beta \mathrm{H}$ immunoreactive protein. ${ }^{3,4,6}$

$\mathrm{pD} \beta \mathrm{H}$ is a highly heritable trait. Abundant evidence indicates that the structural gene encoding the protein, $D B H$, regulates much of the genetic variation in the trait (reviewed by Cubells and Zabetian ${ }^{16}$ ). Prior work has shown several polymorphisms at $D B H$ to be associated with $\mathrm{pD} \beta \mathrm{H}^{7-14}$ These polymorphisms include an insertion/deletion polymorphism located approximately $3 \mathrm{~kb} 5^{\prime}$ to the $\mathrm{DBH}$ transcriptional start site. ${ }^{7}$ Another polymorphism is $-1021 \mathrm{C} \rightarrow \mathrm{T}$ (rs1611115), a single-nucleotide polymorphism (SNP) located approximately $1 \mathrm{~kb}$ upstream of the transcriptional initiation codon of $D B H$ that accounts for $31-52 \%$ of the variance in $\mathrm{pD} \beta \mathrm{H}$ in populations from diverse geographic origins. ${ }^{11,15}$ In European Americans (EAs) and ethnic Germans, rs1611115 resides within a haplotype block (defined as described by Zabetian et al ${ }^{11}$ ) extending from approximately $-2200 \mathrm{bp}$ through intron 4 $(\sim 9.9 \mathrm{~kb})$, but the boundaries of the block remain imprecisely mapped. Rs1611115 accounted for a greater proportion of the variance in $\mathrm{pD} \beta \mathrm{H}$ than any of 10 other common diallelic variants at $D B H$ examined in that study. ${ }^{12}$ Linkage disequilibrium (LD) between each of those variants and rs1611115 appears to be responsible for the association of those variants to $\mathrm{pD} \beta \mathrm{H}^{12}$ Another SNP, rs6271, is located in exon 11 and encodes a nonconservative change in predicted primary amino-acid sequence (arg535cys). Rs6271 is not in LD with rs1611115, and appears to be the only one of four common nonsynonymous SNPs at $D B H$ that independently accounts for additional variance in $\mathrm{pD} \beta \mathrm{H}$ after accounting for the effect of rs1611115. ${ }^{11}$ This observation is consistent with computer modeling suggesting that of the four nonsynonymous SNPs, only rs6271 predicts a substantial change in protein tertiary structure. ${ }^{16}$ Besides the above three polymorphisms, another SNP, rs2519152, located in intron 5 of $\mathrm{DBH}$ and easily genotyped by restriction digest with the endonuclease Taq $\mathrm{I}^{17,18}$ is also of interest because several studies have observed nominally significant associations between this SNP and attention-deficit/hyperactivity disorder (ADHD). ${ }^{19-21}$ In addition, this SNP was found to associate significantly with $\mathrm{pD} \beta \mathrm{H}$ in EAs, even after accounting for the associations of rs1611115 and rs6251 to the phenotype. ${ }^{14}$

Although we have previously demonstrated a robust association between rs1611115 and $\mathrm{pD} \beta \mathrm{H}$ in African Americans (AAs), ${ }^{11}$ data on independent associations of other variants with $\mathrm{pD} \beta \mathrm{H}$ in AAs have not been reported. This paper investigates the association between the four polymorphisms with $\mathrm{pD} \beta \mathrm{H}$, the haplotype and LD structure of $D B H$ in $\mathrm{AA}$ subjects and the possible specific haplotype associated with $\mathrm{pD} \beta \mathrm{H}$. We examined LD among the four SNPs, and used stepwise regression to evaluate whether polymorphisms other than rs1611115 account for significant variance in $\mathrm{pD} \beta \mathrm{H}$ after controlling for the effects of rs1611115.

\section{Methods and materials Samples}

The samples for this study were 109 self-identified AA subjects, recruited in the New Haven, CT area, who met criteria for a variety of substance use or psychiatric disorders. As discussed elsewhere, it is unlikely that differences in psychiatric diagnosis or drug use ${ }^{9}$ substantially alter the relationship between $D B H$ genotype and $\mathrm{pD} \beta \mathrm{H}$. Some of the participants had psychiatric symptoms at the time of assessment, but none were impaired to the point of being unable to provide informed consent for genetic studies, as approved by the Human Investigations Committee of Yale University, or the Human Subjects Subcommittee of the VA Connecticut Health Care System. On average, the subjects were 40.9 ( $\mathrm{SD}=8.6)$-years old, and $71(65.1 \%)$ were male. Some of the genotypes for rs1611115 and rs6271 (51\% of the sample) had been generated already for a previous study, and the association results for rs1611115 (not for rs6271) were reported previously, $^{11}$ but all of the data for ins/del and rs2519152, about half of the data for rs1611115 and rs6271 are newly reported here. In addition, results from the stepwise regression analyses, as well as the haplotypebased regression analyses, are new to this report.

\section{Biochemical, bioinformatic and molecular analysis} Heparinized plasma was separated from the cellular fraction and assayed for $\mathrm{D} \beta \mathrm{H}$ activity as described in detail elsewhere. $^{9}$

The $D B H$ gene contains 12 exons that span approximately $23 \mathrm{~kb}$ of human chromosome 9 (Figure 1). A schematic representation of the $D B H$ gene, showing the relative positions of the SNPs, is shown as Figure 1 below.

We extracted DNA as described previously. ${ }^{22}$ Genotypes at rs2519152 were determined by PCR amplification followed by digestion with Taq I and agarose gel electrophoresis, as described elsewhere. ${ }^{19}$ The primers were: $5^{\prime}$ CTGTATTTG GAACTTGGCATC-3' (forward) and 5'-AGG CATTTTACTACCCAGAGG-3' (reverse). Methods for genotyping the other SNPs have been published previously $7,9,11$ and are available from the corresponding author (JFC).

\section{Statistical analysis}

LD measures $D^{\prime}$ and $r^{2}$ were calculated for all possible combinations of the SNPs analyzed using Haploview. ${ }^{23}$

We used analyses of variance (ANOVAs) to examine the individual effects of the four SNPs on $\mathrm{pD} \beta \mathrm{H}$ and used stepwise linear regression to consider the joint effects of 


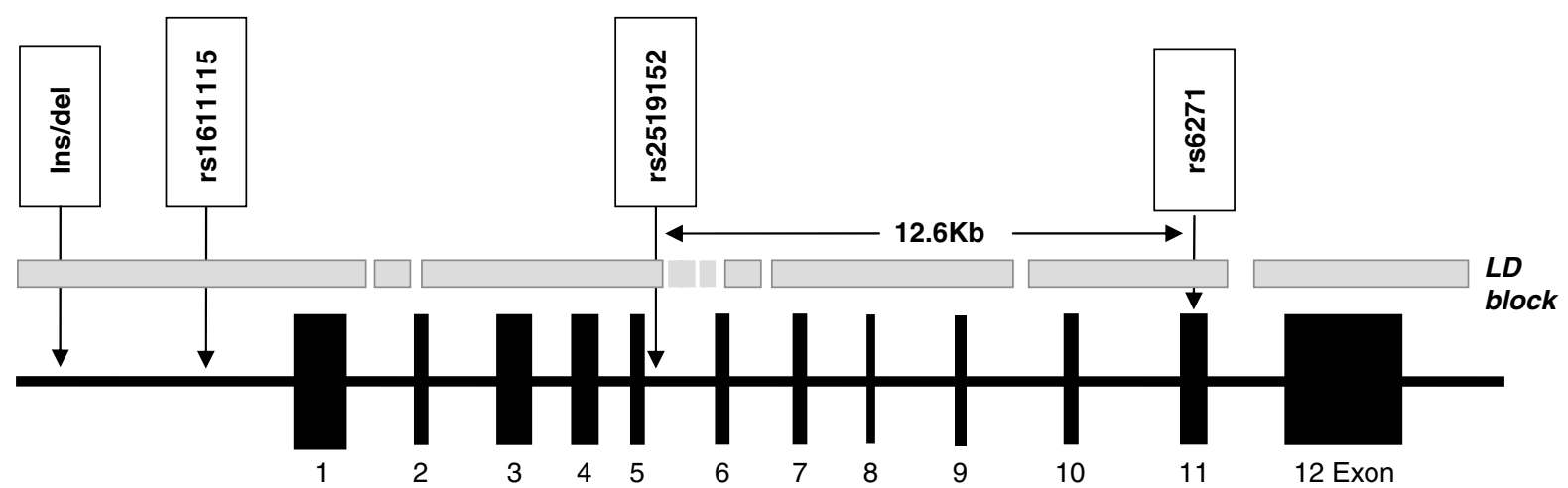

Figure 1 Schematic representation of the DBH gene located on human chromosome $9 q 34$. ins/del, insertion/deletion of $19 \mathrm{bp}$. The LD structure in Yoruban population from HapMap database, as analyzed by the SNP Browser, is also shown as gray blocks.

Table 1 Association of individual diallelic polymorphisms at $D B H$ to plasma $\mathrm{D} \beta \mathrm{H}$ activity

\begin{tabular}{lcccc}
\hline Genotype & Ins/del & $r s 1611115$ & $r s 2519152$ & $r s 6271^{\mathrm{a}}$ \\
\hline 1 & $5.65 \pm 2.24$ (ins/ins $=28)$ & $1.55 \pm 1.23(\mathrm{TT}=8)$ & $5.47 \pm 1.83(\mathrm{CC}=17)$ & - \\
2 & $4.45 \pm 1.90$ (ins/del=64) & $3.71 \pm 1.44(\mathrm{CT}=28)$ & $5.33 \pm 1.87(\mathrm{CT}=41)$ & $7.22 \pm 1.80(\mathrm{CT}=6)$ \\
3 & $3.81 \pm 1.76$ (del/del $=17)$ & $5.36 \pm 1.91(\mathrm{CC}=73)$ & $3.85 \pm 2.05(\mathrm{TT}=51)$ & $4.54 \pm 2.00(\mathrm{CC}=103)$ \\
ANOVA & $F(2,106)=5.52$ & $F(2,106)=22.19$ & $F(2,106)=8.32$ & $F(1,107)=10.50$ \\
Variance explained $\left(R^{2}\right)^{\mathrm{b}}$ & $P=0.007$ & $P=8.9 \times 10^{-9}$ & $P=0.0004$ & $P=0.002$ \\
\hline
\end{tabular}

Abbreviation: ANOVA, analysis of variance.

${ }^{a}$ There were no subjects with TT genotype at this locus.

${ }^{\mathrm{b}}$ After adjustment for age and sex.

these polymorphisms on the trait. Before analysis, we transformed $\mathrm{pD} \beta \mathrm{H}$ to follow an approximate normal distribution using a square-root transformation. ${ }^{11,24}$ Square-root $\mathrm{pD} \beta \mathrm{H}$ was therefore the dependent variable, and genotypes at the DBH SNPs the independent variables. For the stepwise regression procedures, we tested the incremental contribution of rs2519152, rs6271 and ins/ del over and above rs1611115 in explaining variance in square-root $\mathrm{pD} \beta \mathrm{H}$. We explicitly tested the contributions of the SNPs in the following order: rs1611115, rs6271, and rs2519152 and ins/del, because our previous work demonstrated the independent contribution of rs1611115 and rs6271 to variance in $\mathrm{D} \beta \mathrm{H}$ activity in samples from EAs. ${ }^{11,13}$

For haplotype analysis, we used a variation of linear regression ${ }^{25}$ implemented in the WHAP program $^{26}$ to estimate haplotype frequencies and to assess the effects of specific SNP-based haplotypes on the square root of $\mathrm{pD} \beta \mathrm{H}$. Proper inference required the use of an EM algorithm to accommodate the haplotype ambiguity in the genotype data. We established empirical significance of these haplotype effects using permutation procedures.

\section{Results}

Hardy-Weinberg Equilibrium (HWE) was evaluated for each SNP using the website based analysis from the website of Human Genetics, Munich (ihg.gsf.de). No genotypic proportions deviated significantly from HWE (all $P>0.047$ ).

\section{$\mathrm{pD} \beta \mathrm{H}$ associates with genotypes at three different DBH SNPs}

The association of each individual polymorphism to square-root plasma $\mathrm{D} \beta \mathrm{H}$ activity (mean $\pm \mathrm{SD}$; numbers of subjects with each genotype given in parentheses) is shown in Table 1. Because some of the data for rs 161115 were reported earlier, ${ }^{11}$ we also examined the association of rs 161115 to $\mathrm{pD} \beta \mathrm{H}$ just in the 55 new samples. Consistent with the results from analysis of the combined group $(N=109)$, and with the original data reported by Zabetian et al. ${ }^{11}$ There was a significant effect of genotype $(F=7.035$, $P=0.002)$ in the new sample: Subjects with CC had the highest square-root plasma $\mathrm{D} \beta \mathrm{H}$ activity $(N=36$, $4.56 \pm 1.65)$, followed by subjects with CT $(N=14$, $3.96 \pm 1.61)$ and TT $(N=5,1.35 \pm 0.60)$. Comparisons of mean square-root $\mathrm{pD} \beta \mathrm{H}$ within genotype group from the original and new samples showed no significant differences (all $P>0.2$ ). To maximize statistical power, the remaining analyses were therefore performed in the combined sample. As seen in Table 1, each polymorphism was by itself significantly associated with square-root $\mathrm{pD} \beta \mathrm{H}$, with the proportions of variance explained (as indexed by $R^{2}$ ) ranging from 0.065 to 0.29 . Consistent with earlier 
findings, ${ }^{12}$ rs1611115 explained the greatest proportion of the variance.

\section{Linkage disequilibrium analyses and haplotypes}

We used the program Haploview ${ }^{23}$ to calculate the LD measures $D^{\prime}$ and $r^{2}$ for all possible combinations of the SNPs. The results are shown in Figure 2. There was weak LD between rs1611115 and ins/del measured by $D^{\prime}(0.54)$, but no LD was observed among other polymorphisms; there was no substantial LD between any two polymorphisms as measured by $r^{2}$ (all $r^{2}<0.008$ ).

\section{Simultaneous analysis of the contributions of all 4 SNPs}

We performed a stepwise linear regression by using square root of $\mathrm{pD} \beta \mathrm{H}$ as the dependent variable, with age and sex

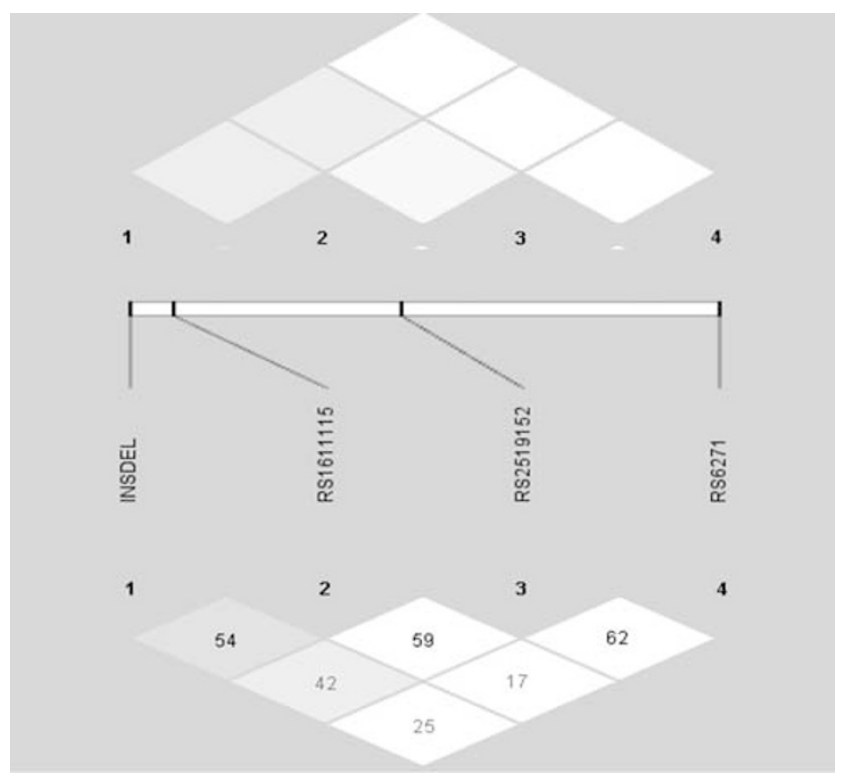

Figure 2 Pairwise LD among five polymorphisms in AA subjects measured by $r^{2}$ (above) and $D^{\prime}$ (below). There was weak LD between ins/del and rs1611115 measured by $D^{\prime}$, but no LD observed among other polymorphisms; there was no LD between any two polymorphisms observed by $r^{2}$. as covariates. Genotypes at rs1611115, rs2519152, rs6271 and ins/del, respectively, were then introduced into the model (coded in additive model) in that order, as suggested by previous results in EAs. ${ }^{11,13,14}$ As shown in Table 2, three out of the four polymorphisms remained in the models, with rs1611115 explaining the largest proportion of variance (28.7\%), and rs2519152 and rs6271 independently accounting for additional variance in $\mathrm{pD} \beta \mathrm{H}(7.5$ and $2.4 \%$, respectively). The maximum value of the collinearity diagnostic parameter, variance inflation factor (VIF) was 1.095 in model 3, suggesting there was minimal collinearity among the three polymorphisms that entered the model.

\section{Three-SNP haplotype analyses}

We used the program WHAP $^{26}$ to calculate the haplotype frequencies of the three SNPs (rs1611115, rs2519152 and rs6271) because ins/del did not have an independent effect on $\mathrm{pD} \beta \mathrm{H}$. As shown in Table 3, the most common haplotype is C-C-C $(\sim 47 \%)$, followed by C-T-C $(\sim 31 \%)$ and T-C-C $(\sim 18 \%)$. Those three haplotypes account for $\sim 94 \%$ of the observed haplotypes and only one of them carries the low $\mathrm{D} \beta \mathrm{H}$ activity associated $\mathrm{T}$ allele at rs1611115. Table 3 also shows that the haplotypes C-T-C and T-C-C, were significantly associated with $\mathrm{pD} \beta \mathrm{H}$ $(P=0.0025$ and 0.0036 , respectively).

Table 3 Haplotype analyses of square root of $\mathrm{pD} \beta \mathrm{H}$ in $\mathrm{AA}$ subjects $\Delta$

\begin{tabular}{lcc}
\hline $\begin{array}{l}\text { Haplotype rs1611115- } \\
\text { rs2519152-rs6271 }\end{array}$ & Frequencies & $\begin{array}{c}\text { Association with } \\
p D \beta H(P)\end{array}$ \\
\hline C-C-C & 0.474 & - \\
C-T-C & 0.306 & 0.0025 \\
T-C-C & 0.176 & 0.0036 \\
T-T-C & 0.029 & 0.209 \\
C-T-T & 0.015 & 0.246 \\
\hline
\end{tabular}

$\triangle$ Data were analyzed by the program WHAP (URL: http://pngu.mgh. harvard.edu/ purcell/whap/). All $P$-values were based on 10000 permutations

Table 2 Model Summary of linear regression using stepwise model

\begin{tabular}{lcccccccccc}
\hline Model & $R$ & $R^{2}$ & Adjusted $R^{2}$ & SE of the estimate & \multicolumn{4}{c}{ Change statistics } \\
& & & & & & $R^{2}$ change & $F$ change & d.f. 1 & d.f.2 & Significant $F$ change \\
\hline 1 & $0.541^{\mathrm{a}}$ & 0.293 & 0.287 & 1.75859 & 0.293 & 44.373 & 1 & 107 & $1.2 \times 10^{-9}$ \\
2 & $0.611^{\mathrm{b}}$ & 0.374 & 0.362 & 1.66285 & 0.081 & 13.676 & 1 & 106 & 0.0003 & 1.000 \\
3 & $0.635^{\mathrm{c}}$ & 0.403 & 0.386 & 1.63095 & 0.029 & 5.187 & 1 & 105 & 0.025 & $1.012-1.095$ \\
\hline
\end{tabular}

Abbreviations: d.f., degrees of freedom; SE, standard error.

apredictors: (constant), genotype at rs1611115.

bPredictors: (constant), genotype at rs1611115, rs6271.

CPredictors: (constant), genotype at rs1611115, rs6271 and rs2519152.

VIF, variance inflation factor. 


\section{Discussion}

This study examined the relationship between DBH gene polymorphisms and $\mathrm{pD} \beta \mathrm{H}$ in AAs more closely than our prior study, ${ }^{11}$ in a larger set of samples. Our results, both in the combined group $(N=109)$ and in the group of new samples $(N=55)$, replicated the association between SNP rs 1611115 and $\mathrm{pD} \beta \mathrm{H}$ reported in the prior AA sample. ${ }^{11}$ Results from analysis of additional polymorphisms showed apparently significant associations between $\mathrm{pD} \beta \mathrm{H}$ and each of the three polymorphisms, including a 19-bp insertion/deletion and three SNPs. However, the failure of the former polymorphism to remain in the regression model after controlling for other genotypes strongly suggests the apparent difference in activity associated with this variant can be explained entirely by LD to rs 1611115 .

As in subjects of European origin, ${ }^{11,13,14}$ rs1611115 explained the largest proportion of variance in $\mathrm{pD} \beta \mathrm{H}$, However, as we reported previously, ${ }^{11}$ rs1611115 accounts for a lower proportion of the variance in $\mathrm{pD} \beta \mathrm{H}$ than in subjects of European origin. Also in accordance with previous results from EAs, ${ }^{14}$ rs2519152 and rs6271 appear to account for additional variance in $\mathrm{pD} \beta \mathrm{H}$ independent of rs1611115. Finally, the data reported here are the first to examine the haplotype structure of $D B H$ in an AA sample. It should be noted, however, that more detailed studies are necessary, as the variants examined do not tag all the common variation at $D B H$ present in AAs.

Substantial differences in allele and haplotype frequencies often occur across populations of differing geographic origins. ${ }^{27}$ Although such differences can complicate efforts to identify causal polymorphisms for specific phenotypes, consistency in the pattern of association between genotype and phenotype in different populations can validate genotype-phenotype associations. We found that both allele and haplotype frequencies of the variants examined here differ in AAs and those of European origin.7,9,11-14 For example, the minor allele frequency (MAF) for $\mathrm{C}$ of rs2519152 in this sample was $34.8 \%$ (based on Table 1), whereas it was $42.8 \%$ in EAs as reported by Tang et al. ${ }^{14}$ In addition, rs2519152 was in stronger LD with rs1611115 and rs6271 in EAs than in AA, especially as measured by $r^{2}$ $\left(D^{\prime}=0.59\right.$ and 0.62 , respectively; $r^{2}=0.04$ and 0.019 , respectively). That lower degree of LD is consistent with the $D B H$ LD structure apparent in the data from Yorubans in the HapMap database (www.hapmap.org). Comparisons of allele and haplotype frequencies in other samples of AAs and EAs in our lab further support differences in allele and haplotype frequencies in the two populations: among 31 SNPs typed across $D B H$ and a neighboring gene, SARDH, 13 showed significantly different allele frequencies in AA and EA (Y.-L. Tang, E.B. Binder and J.F. Cubells (2006), unpublished data), including rs6271 $(P=0.000029)$. Despite these clear differences in allele and haplotype frequencies, comparison of the patterns of genotype$\mathrm{pD} \beta \mathrm{H}$ associations in the two populations reveal similar patterns. In addition, the direction of genotype $-\mathrm{pD} \beta \mathrm{H}$ associations at rs1611115, the major contributor to the variance of $\mathrm{pD} \beta \mathrm{H}$, was identical in both populations, so that the same allele associated with higher or lower $\mathrm{pD} \beta \mathrm{H}$ in both analyses.

In summary, we conclude that: (1) in AA and EA subjects, there are similar associations between $D B H$ polymorphisms and $\mathrm{pD} \beta \mathrm{H} ;(2)$ two common haplotypes, C-T-C and T-C-C are significantly associated with $\mathrm{pD} \beta \mathrm{H}$ in $\mathrm{AA}$; (3) the association of ins/del with $\mathrm{pD} \beta \mathrm{H}$ reflects its $\mathrm{LD}$ with rs1611115; but (4) the other two SNPs (rs6271 and rs2519152) appear to contribute small but independent effects on $\mathrm{pD} \beta \mathrm{H}$. Further work is necessary to determine whether the significantly associated SNPs studied here are functional, or in LD with other functional variants. Clearly, analyses of association between SNPs at $D B H$ and clinical phenotypes requires analysis of multiple SNPs across the locus.

\section{Acknowledgements}

This work was supported by a NIDA INVEST fellowship (to YLT), KO2 DA015766 and R01 DA12422 (to JFC), K08 NS044138 (to CPZ). The authors are grateful to Laura Heizman and Matthew Girgenti for their expert technical assistance, and to all the study participants for their donation of blood for analysis.

\section{References}

1 Weinshilboum RM: Human biochemical genetics of plasma dopamine-beta-hydroxylase and erythrocyte catechol-o-methyltransferase. Hum Genet Suppl 1978; 101-112.

2 O'Connor DT, Cervenka JH, Stone RA et al: Dopamine betahydroxylase immunoreactivity in human cerebrospinal fluid: properties, relationship to central noradrenergic neuronal activity and variation in Parkinson's disease and congenital dopamine beta-hydroxylase deficiency. Clin Sci (London) 1994; 86: 149-158.

3 O'Connor DT, Levine GL, Frigon RP: Homologous radio-immunoassay of human plasma dopamine-beta-hydroxylase: analysis of homospecific activity, circulating plasma pool and intergroup differences based on race, blood pressure and cardiac function. J Hypertens 1983; 1: 227-233.

4 Dunnette J, Weinshilboum R: Human serum dopamine betahydroxylase: correlation of enzymatic activity with immunoreactive protein in genetically defined samples. Am J Hum Genet 1976; 28: $155-166$

5 Oxenstierna G, Edman G, Iselius L, Oreland L, Ross SB, Sedvall G: Concentrations of monoamine metabolites in the cerebrospinal fluid of twins and unrelated individuals - a genetic study. J Psychiatr Res 1986; 20: 19-29.

6 Ebstein RP, Park DH, Freedman LS, Levitz SM, Ouchi T, Goldstein M: A radioimmunoassay of human circulatory dopamine-betahydroxylase. Life Sci 1973; 13: 769-784.

7 Cubells JF, Kranzler HR, McCance-Katz E et al: A haplotype at the DBH locus, associated with low plasma dopamine beta-hydroxylase activity, also associates with cocaine-induced paranoia. Mol Psychiatry 2000; 5: 56-63.

8 Cubells JF, Price LH, Meyers BS et al: Genotype-controlled analysis of plasma dopamine beta-hydroxylase activity in psychotic unipolar major depression. Biol Psychiatry 2002; 51: 358-364.

9 Cubells JF, van Kammen DP, Kelley ME et al: Dopamine betahydroxylase: two polymorphisms in linkage disequilibrium at the 
structural gene DBH associate with biochemical phenotypic variation. Hum Genet 1998; 102: 533-540.

10 Wei J, Ramchand CN, Hemmings GP: Possible control of dopamine beta-hydroxylase via a codominant mechanism associated with the polymorphic (GT)n repeat at its gene locus in healthy individuals. Hum Genet 1997; 99: 52-55.

11 Zabetian CP, Anderson GM, Buxbaum SG et al: A quantitativetrait analysis of human plasma-dopamine beta-hydroxylase activity: evidence for a major functional polymorphism at the DBH locus. Am J Hum Genet 2001; 68: 515-522.

12 Zabetian CP, Buxbaum SG, Elston RC et al: The structure of linkage disequilibrium at the DBH locus strongly influences the magnitude of association between diallelic markers and plasma dopamine beta-hydroxylase activity. Am J Hum Genet 2003; 72: 1389-1400.

13 Tang Y, Anderson GM, Zabetian CP, Kohnke MD, Cubells JF: Haplotype-controlled analysis of the association of a nonsynonymous single nucleotide polymorphism at DBH $(+1603 \mathrm{C}$ $->$ T) with plasma dopamine beta-hydroxylase activity. Am J Med Genet B Neuropsychiatr Genet 2005; 139: 88-90.

14 Tang Y, Buxbaum SG, Waldman I et al: A single nucleotide polymorphism at $\mathrm{DBH}$, possibly associated with attention-deficit/ hyperactivity disorder, associates with lower plasma dopamine beta-hydroxylase activity and is in linkage disequilibrium with two putative functional single nucleotide polymorphisms. Biol Psychiatry 2006; 60: 1034-1038.

15 Kohnke MD, Zabetian CP, Anderson GM et al: A genotype-controlled analysis of plasma dopamine beta-hydroxylase in healthy and alcoholic subjects: evidence for alcohol-related differences in noradrenergic function. Biol Psychiatry 2002; 52: 1151-1158.

16 Cubells JF, Zabetian CP: Human genetics of plasma dopamine beta-hydroxylase activity: applications to research in psychiatry and neurology. Psychopharmacology (Berl) 2004; 174: $463-476$.
17 D'Amato T, Leboyer M, Malafosse A et al: Two TaqI dimorphic sites at the human beta-hydroxylase locus. Nucleic Acids Res 1989; 17: 5871.

18 Wu S, Muhleman D, Comings D: PCR amplification of the Taq I B1/B2 polymorphism at intron 5 of the dopamine beta-hydroxylase gene. Psychiatr Genet 1997; 7: 39-40.

19 Daly G, Hawi Z, Fitzgerald M, Gill M: Mapping susceptibility loci in attention deficit hyperactivity disorder: preferential transmission of parental alleles at DAT1, DBH and DRD5 to affected children. Mol Psychiatry 1999; 4: 192-196.

20 Roman T, Schmitz M, Polanczyk GV, Eizirik M, Rohde LA, Hutz $\mathrm{MH}$ : Further evidence for the association between attentiondeficit/hyperactivity disorder and the dopamine-beta-hydroxylase gene. Am J Med Genet 2002; 114: 154-158.

21 Smith KM, Daly M, Fischer M et al: Association of the dopamine beta hydroxylase gene with attention deficit hyperactivity disorder: genetic analysis of the Milwaukee longitudinal study. Am J Med Genet 2003; 119B: 77-85.

22 Cubells JF, Kobayashi K, Nagatsu T et al: Population genetics of a functional variant of the dopamine beta-hydroxylase gene (DBH). Am J Med Genet 1997; 74: 374-379.

23 Barrett JC, Fry B, Maller J, Daly MJ: Haploview: analysis and visualization of LD and haplotype maps. Bioinformatics 2005; 21: $263-265$.

24 Wilson AF, Elston RC, Siervogel RM, Tran LD: Linkage of a gene regulating dopamine-beta-hydroxylase activity and the $\mathrm{ABO}$ blood group locus. Am J Hum Genet 1988; 42: 160-166.

25 Lake SL, Lyon $\mathrm{H}$, Tantisira $\mathrm{K}$ et al: Estimation and tests of haplotype-environment interaction when linkage phase is ambiguous. Hum Hered 2003; 55: 56-65.

26 Purcell S, Sham P: WHAP (haplotype-based association analysis package). Cambridge, MA

27 The International HapMap Consortium: A haplotype map of the human genome. Nature 2005; 437: 1299-1320. 